\title{
Galactic origin of cosmic rays
}

\author{
Measurements of $\gamma$-rays from the Small Magellanic Cloud seem to have ruled out the possibility that cosmic rays \\ are uniformly spread through extragalactic space, but do not say much more about their origin.
}

So that's it then. Cosmic rays are not a universal phenomenon, spread through intergalactic space as well as other galaxies, but those that it is possible to observe near the surface of the Earth are predominantly the products of this Galaxy alone and, apart from a little leakage at the edges, are confined to it.

Declarative statements such as that, on such great issues, cannot often be made these days. As will become apparent, even this can bear a little qualification. Direct proof of any such assertion naturally lies a long way off; there are as yet no plans for launching intergalactic measuring devices, for example. But may it not be possible to tell something about the general distribution of cosmic rays by looking for evidence of their presence (or absence) in other galaxies?

The obvious difficulty is that the other galaxies are so far away. The more serious difficulty is to know how it would be possible to tell whether there are cosmic rays in other galaxies. Searching for cosmogenic carbon or some isotopic consequence of a flux of cosmic rays comparable with that at the Earth will remain a hopeless task, at least until somebody finds an extragalactic (or, for that matter, a galactic) Earth-like planet. Some other chain of inference is called for.

The first clear recipe for deciding between a galactic and metagalactic origin for cosmic rays was provided more than 20 years ago by V. L. Ginzburg (who is still attached to the Lebedev Institute in Moscow). In 1972, he suggested (while disclosing a prejudice in favour of the galactic hypothesis) that a measurement of $\gamma$-rays from the Large and Small Magellanic Clouds would provide a crucial distinction between the two possibilities (Nature Physical Science 239, 8; 1972).

How does the argument go? The energetic particles of cosmic rays inevitably collide with interstellar gas, producing energetic $\gamma$-rays. But the quantities of interstellar matter in the Magellanic Clouds had already been inferred from measurements by radioastronomers of neutral hydrogen in them. Ginzburg then simply calculated the flux of $\gamma$-rays expected from the two satellite galaxies on the two hypotheses.

On the metagalactic view, the $\gamma$-ray flux should be that produced by a flux of cosmic rays similar to that near the Earth; otherwise, it would be smaller. Restricting the argument to $\gamma$-rays more energetic than $100 \mathrm{MeV}$ (to avoid low-energy stellar processes), Ginzburg concluded that, if his preferred NATURE · VOL 361 - 21 JANUARY 1993 galactic hypothesis were correct, the combined $\gamma$-ray flux from the Magellanic Clouds would be only a third of that expected on the metagalactic view - and that the output of the two clouds should also be significantly different.

Ginzburg's expectations have now been amply confirmed by data gathered by the Compton Gamma Ray Observatory, a satellite launched in 1991 from the United States and managed from the Goddard Space Flight Center. The essence of the instrument is a $\gamma$-ray telescope built so as to detect $\gamma$-rays much as that job was done in the old cloud-chamber days, by using parallel sheets of a material such as lead to convert them into electron-positron pairs. The telescope provides information about the direction of the $\gamma$-rays received as well as their energy. Calibration has shown that the background noise is at least an order of magnitude less than the signal expected from intergalactic space.

The inference that cosmic rays have a galactic origin (and that the Magellanic clouds produce their own), or at least that the metagalactic hypothesis does not stand up, is based on six weeks worth of data gathered when the telescope was pointing towards the Small Magellanic Cloud (Streekumar, P., et al., Phys. Rev. Lett. 70, 127; 11 January 1993). The authors, of whom there are 16 (four from the Max Planck Institute of Extraterrestrial Physics at Garching, Munich), have carefully subtracted from the measurements the $\gamma$-ray flux expected from the Galaxy and from the local Compton scattering of low-energy photons by energetic cosmic $\gamma$-ray particles.

The outcome is that the measured flux of $\gamma$-rays more energetic than $100 \mathrm{MeV}$ is reckoned not to be greater than $0.5 .10^{-7}$ photons per square centimetre per second; this estimate allows for the uncertainties of the measurement at a confidence limit of 95 per cent. In other words, the chance that the output from the Small Magellanic Cloud exceeds this limit is 5 per cent. By contrast, on the metagalactic view, the expected flux from the cloud should have been $2.4 \pm$ $0.5 .10^{-7}$ photons $\mathrm{cm}^{-2} \mathrm{~s}^{-1}$. The two numbers differ by a factor of five and, although the error of the estimated output on the metagalactic view may seem uncomfortably large to some, few will doubt that it is large enough to carry the day for the view that cosmic rays have a galactic origin.

So far as it goes, this outcome is comforting enough. Ginzburg, at least, should be pleased, not least for his clear statement 20 years ago that instruments then being designed for satellite use would have sufficient sensitivity to discriminate between the two possibilities. But, inevitably, things have become a little more complicated since 1972, not least because of the doubts astronomers have thrown on the intrinsic stability of the Small Magellanic Cloud.

That something fishy is going on seems well established. Radioastronomy suggests that the satellite galaxy has two components with different velocities, optical observations have suggested that it is thinner along the line of sight than it should be if it is to be stable, while there is a tidal theory involving a close encounter with the Large Cloud less than 100 million years ago that provides a means by which it may have been rendered unstable. These arguments are relevant, because the possibility that the Small Magellanic Cloud may be in the process of disintegrating is the origin of the large uncertainty in the $\gamma$-ray flux expected on the metagalactic view.

For what it is worth, measurements with the same instruments of the Large Magellanic Cloud, believed to be stable enough, yield only an inconclusive result. The measured flux is less than that expected from the metagalactic view, but given the uncertainties of measurement and calculation, the difference is insignificant. There the inferred intensity of cosmic rays, which exert an expansionary (or counter-gravitational) pressure within a stable galaxy, seems comparable with that near us.

Meanwhile, it will have been noted that this development may have ruled out one hypothesis whose implications for the energy balance of the Universe have always made some people uncomfortable, but has not pointed to the true sources of cosmic rays. At least the metagalactic camp has been able to claim that quasars, radio galaxies and other distant but poorly understood objects were plausible sources of energetic particles. If the major sources of energetic particles must now be sought within the Galaxy, there will be some difficulty in adding up the effects of supernovae, collapsing binaries, $\gamma$-ray bursters and the like to yield the observed flux of cosmic rays. And while Fermi's mechanism of the acceleration of low-energy particles to cosmic $\gamma$-ray energy by random magnetic fields remains a possibility, it has the drawback of being almost untestable.

But people will at least now know where to look.

John Maddox 